\title{
A clinical audit and service evaluation of a new role in primary care: a musculoskeletal practitioner Amanda Hensman-Crook, MSc SRP
}

\section{INTRODUCTION}

With the increasing demands on primary care, it is essential to find a different way of working to manage the ever-growing numbers of patient consultations. It needs to be a system where patients are seen in an acceptable timeframe, managed efficiently, effectively, with excellent care and all within tight financial margins. ${ }^{(1,2)}$ The role of the general practitioner (GP) has changed dramatically over the last decade; the Quality and Outcomes framework, introduced as part of the GP contract in 2004, and the continual need for service evolvement, creates greater pressure on top of their medical role to fulfil the vision.

As well as internal primary care service provision, the need for consideration of relevant referral into secondary care from primary care, to meet the I8-week waiting list target, ${ }^{(3)}$ is of great importance. This matter has been addressed within secondary care for musculoskeletal referrals by triage by 'extended-scope physiotherapists' in orthopaedic clinics with good results. ${ }^{(4)}$ The service has improved the relevance of referrals into musculoskeletal secondary care, reducing waiting lists for orthopaedic surgeons. This role has extended into primary care, ${ }^{(5)}$ but largely through GP referral, or within a 'one-stop clinic' with a multidisciplinary team (MDT) on site, including orthopaedic surgeons. The musculoskeletal practitioner role in primary care is a direct referral service. With patients able to have a self-referral option, it opens more space for GPs to manage medical conditions, with the benefit of shortening the amount of time that the patient has to wait for a consultation.

The musculoskeletal practitioner post was an extension of thought following the highly successful introduction of nurse practitioners into primary care. ${ }^{(6,7), 8)}$ The nurse practitioner service has helped to compliment the GP's load, improve the patient's care pathway, and to provide a first-class service in their area of care.(9) The musculoskeletal practitioner role was created to provide patients with a highly specialised service for the assessment, diagnosis and triage for musculoskeletal problems, ${ }^{(5,10)}$ as well as to address the previously highlighted current demands on the primary and secondary care services.

\section{BACKGROUND}

Aware of the constraints and ever-changing demands on primary care, a GP practice in the South Lakeland locality took the initiative to look at a different way to manage their patient load using the principles of 'Productive General Practice', (II) which supports change in primary care, enabling the creation of capability and capacity to allow service improvement. This can empower practices to implement new roles and care pathways.
Musculoskeletal-related problems make up almost a quarter of a GP's caseload, ${ }^{(12,13,14)}$ taking up significant GP consultation time. Thought was also given to the need for the relevance of referral into secondary care from primary care into orthopaedic clinics. ${ }^{(5,10)}$ With the latter identified, musculoskeletal care was pinpointed as something that could be more effectively streamlined to ensure the best care for this patient group, and to create space for GP consultation for other medical conditions.

A literature search was conducted around orthopaedic triage services in both primary and secondary care. ${ }^{(4,5,10)}$ From the evidence, the strengths of existing posts were adopted, and then discussions were opened around how the role could be further developed to improve efficiency and effectiveness of the role.

Once a job description had been agreed, a three-month trial took place to explore the impact of the position on the practice and patient care before deciding whether or not to establish it into the practice longer term.

\section{Aims of the post}

- to develop, refine and enhance the model of service delivery for musculoskeletal consultations based on patient and other stakeholder perspectives

- to provide direct access to a specialised musculoskeletal service for patients

- to create space in GP consultations to focus on other medical conditions, and to provide greater accessibly to see the GP with a shorter waiting time.

- to ensure relevant musculoskeletal referral into secondary care

- to evaluate the impact of the musculoskeletal practitioner post regarding: accessibility, patient satisfaction, safety, efficiency of the care pathway, cost effectiveness and the impact on the wider health system

- to enhance musculoskeletal knowledge within the MDT

\section{The role}

- clinical musculoskeletal physiotherapist with extendedscope skills: injection therapy; ordering and interpretation of $X$-rays/ultrasound scans/magnetic resonance imaging (MRI) scans and bloods; non-medical prescribing

- provision of highly specialised assessment and diagnostic triage to determine the basis for referral, investigations and further management into secondary care

- musculoskeletal education and advice for MDT

- audit/research 


\section{Population}

All patients presenting to the surgery with a musculoskeletal problem or rheumatological condition are eligible for the service.

Patients gain access to see the musculoskeletal practitioner by:

- booking directly with the musculoskeletal practitioner at reception

- via a GP or nurse practitioner to redirect patients into the clinic if presenting with musculoskeletal issues so can focus on medical problems, book for follow-up for interpretation and explanation of relevant investigations, for steroid injection, or for follow-up after an initial consultation for a specialised assessment and triage

- the onsite physiotherapist for injection, further investigation or a second opinion for unresponsive patients

\section{Method}

Data collected over the three-month audit period determined:

- capacity - the number of patients filling available slots

- source of referral - patient numbers collected for: direct referral; GP; nurse practitioner; physiotherapist; and followups

- outcome of assessment - data collected for: exercise/advice; injection; referral to physiotherapy, consultant, GP; bloods; X-ray; and aspiration

- patient satisfaction survey - based on the validated PSQ18 , $^{(15)}$ directly relating to the musculoskeletal practitioner service regarding: time given for consultation; thoroughness of assessment; explanation of condition given; advice regarding condition; the outcome from the consultation; and the approach of the practitioner to the consultation

\section{DISCUSSION}

\section{Capacity}

The musculoskeletal practitioner clinic was run over two sessions of four hours a week. Over the three-month audit period, a total of 206 patients were seen, with an average of $87 \%$ of available appointments filled. The capacity remained the same in the first two months of the audit at $83 \%$, increasing to $94 \%$ in the third month (see figure I).

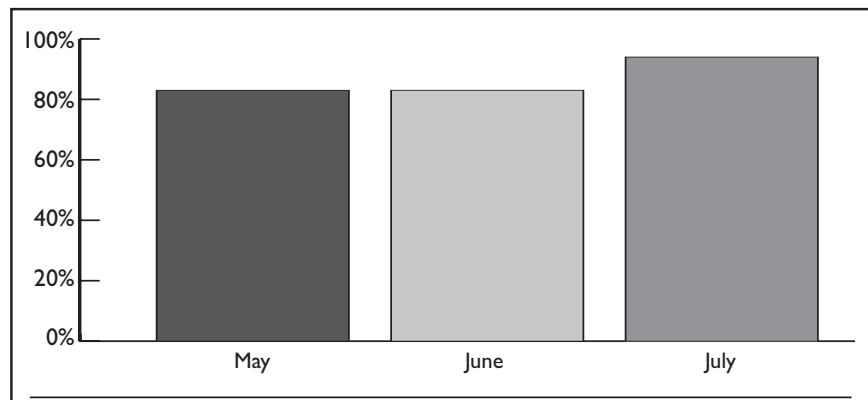

Figure I Capacity (number of available appointments used)

\section{Referral}

Referrals were taken directly at reception, via GP, nurse practitioners, and the onsite physiotherapist (see figure 2). Follow-up consultations by the musculoskeletal practitioner accounted for the rest of the appointments used. The followups were after injection, $\mathrm{X}$-ray, bloods, aspiration or an exercise regime as a one-off before discharge or referral into other services.

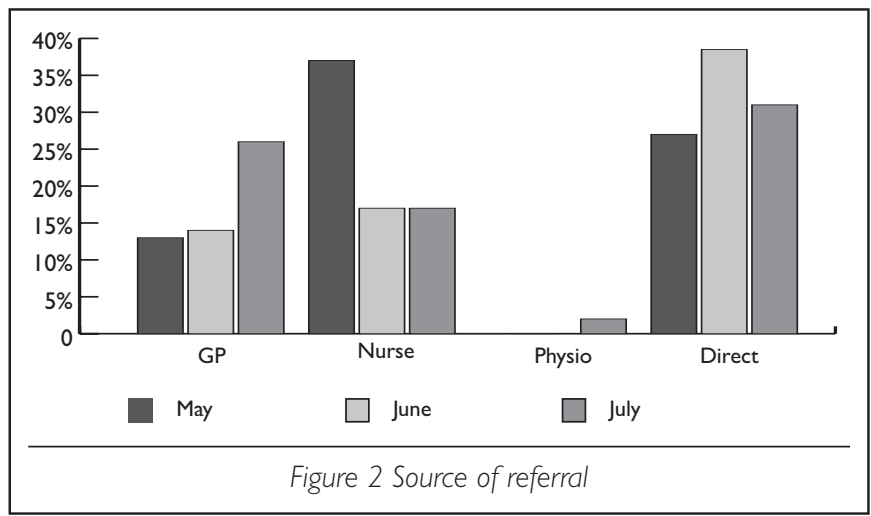

GP referrals increased from $7 \%$ to $22 \%$. Of the patients referred by the GP, 35\% were un-assessed musculoskeletal problems, and $65 \%$ following single assessment for further investigation, post $X$-ray explanation and triage. Over the three months, $81 \%$ of all patients seen had no GP contact, creating 168 available consultation spaces over the threemonth period.

\section{Outcome from assessment}

The average percentage of outcome from assessment or follow-up are (higher to lower percentage): exercise and advice $40 \%$; referral to physiotherapy $27 \%$; injection $20 \%$; X-ray $5 \%$; referral to consultant 5\%; referral to GP, for bloods and aspiration 1\% (see figure 3).

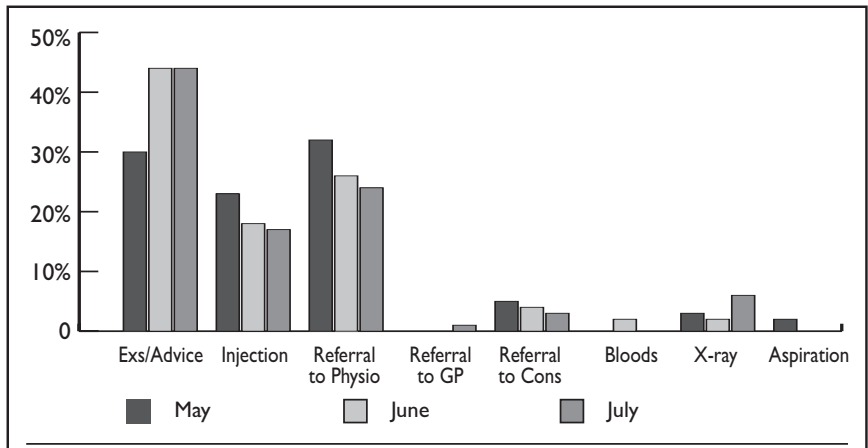

Figure 3 Outcome of assessment

\section{Patient satisfaction}

The survey was handed out to all patients, $50 \%$ of the questionnaires were returned.

Overall patient satisfaction of the service showed: 90\% 'excellent'; $9 \%$ 'very good'; and I\% 'good' (see figure 4).

Each category tested (time given for consultation, thoroughness of examination, explanation given for the complaint, advice for the condition, outcome from consultation and approach the consultation) showed results of: between $88 \%$ and $96 \%$ 'excellent'; $4 \%$ and $12 \%$ very good; and $4 \%$ good in the time given category (see figure 5). 

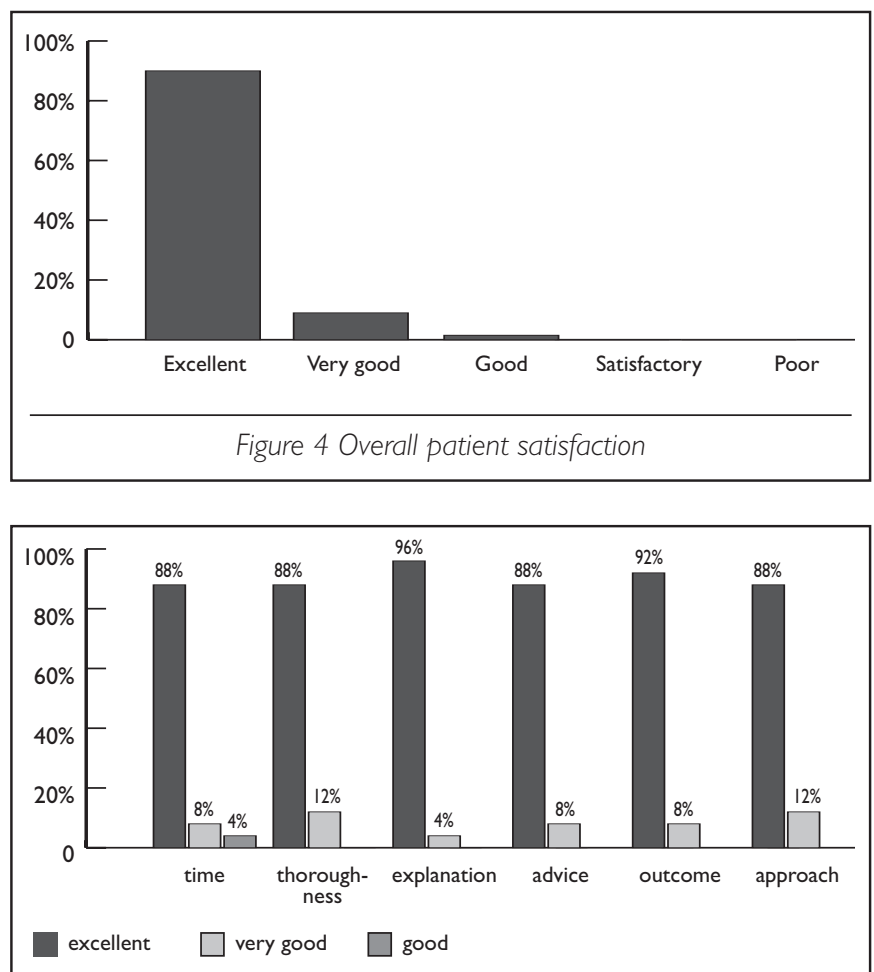

Figure 5 Breakdown of patient satisfaction survey

\section{CONCLUSION}

The musculoskeletal practitioner role has enhanced the model of service delivery for musculoskeletal consultations benefiting the practice, patients, GPs, the wider MDT and secondary care.

It has provided direct access for patients into a specialised area of care, which shortens and improves the quality of their care pathway. The patient has the opportunity for immediate highly specialised musculoskeletal assessment; with an optimal management plan for their condition delivered on consultation. Referral into secondary care is streamlined, and injections are done without a need for further appointments. It has been met with enthusiasm by the patients, with encouraging patient satisfaction results. The initial fear that it would create extra work in the practice has not materialised. It is hypothesised that patients' knowledge of a nurse practitioner role has extended to help understand the role of a musculoskeletal practitioner.

Capacity has been created for GP consultations. This has been possible through direct access into the new service, and by the ability to divert musculoskeletal problems if seen during a consultation into the musculoskeletal clinic. Furthermore, it has provided an opportunity to discuss musculoskeletal cases with an expert in the field, improving knowledge which helps relevant referral into secondary care. This has also benefitted the nurse practitioners on site.

Financially, it has the potential to be cost effective. Further study is required over a longer time period to establish fact. GP are expensive gatekeepers into secondary care relative to a musculoskeletal practitioner. Musculoskeletal problems account for almost a quarter of many practices consultations. . $^{(2,13,14)}$ Depending on the size of a practice, the amount of hours required to harness these patients will differ. Costs can be further reduced in small practices by sharing a musculoskeletal practitioner to provide clinics in a locality. On a wider scale, there is a positive financial implication for secondary care through accurate referral. With relevant referral, throughput can be expatiated, shortening waiting times for surgery with the capacity to increase income generation from joint replacement.

The musculoskeletal practitioner role has provided an effective, efficient and a potentially financially viable role within primary care. It addresses both primary and secondary care service requirements, as well as providing an easily accessible, highly specialised musculoskeletal service for patients.

\section{REFERENCES}

I. McElduff P, Lyratzopoulos G, Edwards R, Heller RF, Shekelle P, Roland $M$. Will changes in primary care improve health outcomes? Modelling the impact of financial incentives introduced to improve quality of care in the UK. Qual Saf Health Care 2004; I3(3): 191-7

2. Roland M, Campbell S, Bailey N, Whalley D, Sibbald B. Financial incentives to improve the quality of primary care in the UK: predicting the consequences of change. Prim Health Res Dev 2006;7(1): 18-26

3. Lewis R, Abbleby J. Can the English NHS meet the 18 week waiting list target? JRSM 2006; 99( I): 10-13

4. Sephton R, Hough E. Evaluation of a primary care musculoskeletal clinical assessment service: a preliminary study. Physiother 2010;96(4):296-302

5. Hattam P, Smeatham A. Evaluation of an orthopaedic screening service in primary care. Br J Clin Gov 1999:4(2):45-9

6. Horrocks S, Anderson E, Salisbury C. Systematic review of whether nurse practitioners working in primary care can provide equivalent care to doctors. Br Med J 2002;324(734I):819

7. Kinnersley P, Anderson E, Parry K, et al. Randomised controlled trial of nurse practitioner versus general practitioner care for patients requesting 'same day' consultations in primary care. Br Med J 2000;320(724I): 1043

8. Mundinger MO, Kane RL, Lenz ER, et al. Primary outcomes in patients treated by nurse practitioners or physicians: a randomised trial. JAMA 2000;283(I):59-68

9. Venning P, Durie A, Roland M, Roberts C, Leese B. Randomised controlled trial comparing cost effectiveness of general practitioners and nurse practitioners in primary care. Br Med J 2000:320(724I): 1048

10. Samsson K, Larsson ME. Physiotherapy screening of patients referred for orthopaedic consultation in primary healthcare-a randomised controlled trial. Med J Aust 2007; I 86( I 2):625-8

I I. NHS. Institute for Innovation and Improvement. www.institute.nhs.uk

12. Pekka M, Esko K, Ahonen R, et al. Pain as a reason to visit the doctor: a study in Finnish primary care. Pain 200 I;89(2): 175-80

13. Rekola K, Keinanen-Kiukaanniemi S, Takala J. Use of primary health services in sparsely populated country districts by patients with musculoskeletal symptoms: consultation with a physician. J Epidemiol Community Health 1993;47(2): 153-7

14. Urwin M, Symmons D, Allison T, et al. Estimating the burden of muskulosketal disorders in the community: the comparative prevalence of symptoms at different anatomical sites, and the relation to social deprivation. Ann Rheum Dis 1998;57(I I):649-55

15. Grogan S, Conner M, Norman P,Willits D, Porter I. Validation of a questionnaire measuring patient satisfaction with practitioner services. Qual Health Care 2000;9(4):210-5 